\title{
Role of the prefrontal cortex in the neonatal ventral hippocampus lesion, an animal model of schizophrenia
}

\author{
Gonzalo Flores' and Julio Cesar Morales-Medina ${ }^{2}$ \\ 'Instituto de Fisiología, Benemérita Universidad Autónoma de Puebla. 14 Sur 6301, Puebla, México \\ ${ }^{2}$ Centro de Investigación en Reproducción Animal, CINVESTAV- Universidad Autónoma de Tlaxcala, AP 62, CP 90000, Tlaxcala, México
}

\section{Article Info}

\section{Article Notes}

Received: May 05, 2016

Accepted: June 23, 2016

\section{*Correspondence:}

Dr. Gonzalo Flores

Laboratorio de Neuropsiquiatría

Instituto de Fisiología, Benemérita Universidad Autónoma de

Puebla,

14 Sur 6301, CP. 72570, Puebla, México Telephone: (522)

2295500 ext. 7322

Email: gonzaloflores56@gmail.com;

gonzalo.flores@correo.buap.mx

(c) 2016 Flores $\mathrm{G}$. This article is distributed under the terms of the Creative Commons Attribution 4.0 International License

\section{Keywords}

Schizophrenia

Prefrontal cortex

Connectivity

Neonatal ventral hippocampal lesion

\section{ABSTRACT}

Schizophrenia is a complex mental disorder that starts at early adulthood with a combination of positive and negative symptoms as well as cognitive impairments. It is well known that dendritic spine density and dendritic length of the pyramidal neurons of the prefrontal cortex (PFC) are reduced in the postmortem tissue of schizophrenia patients. In addition, the volume of the PFC is reduced in this mental disorder. A possible hypothesis for these morphological changes suggests that the disruption between PFC and hippocampus, at an early age is involved in the pathophysiology of schizophrenia. Furthermore, rats with bilateral lesion of the neonatal ventral hippocampus ( $\mathrm{VVHL}$ ) at an early age is an example of the initial disruption between hippocampus and PFC and also exhibits a reduction in the synaptic connections in the PFC. The present mini-review discusses the neurochemical and morphological changes in the PFC of rats that underwent $\mathrm{nVHL}$, an animal model of schizophrenia.

\section{Introduction}

Schizophrenia is a mental disorder (DSM-5) that affects approximately $1 \%$ of the world population. This disorder is not only devastating for the patient but also affects the family ${ }^{1}$. Its prognosis depends on the number of psychotic outbreaks, progression of cognitive impairments and response to neuroleptics. This psychotic disorder is characterized by the presence of symptoms classified as positive (increased locomotion in response to psychostimulants, hallucinations, delusions and thought disorders), negative (deficits in social interaction, anhedonia, and affective flattening, among others) and cognitive deficits (attention and memory deficits). In recent years, with the advancement in the image analysis and microscope systems, the neural morphological studies in postmortem tissue from schizophrenic patients have shown constant and critical changes in the prefrontal cortex (PFC), which may explain some of the symptoms observed in this complex disorder ${ }^{2}$. Moreover several animal models of schizophrenia have been developed over the past 20 years. Although there is no animal model that can replicate all aspects of complexity that occurs in schizophrenia, but they can correlate changes in the animal behavior with the classification of the symptoms of schizophrenia. An increased expression of a behavior is a positive symptom, such as increased locomotor activity in a new environment. Negative symptoms involve reduction in certain behaviors, such as grooming. In addition, some behaviors may relate directly, such as deficits in social interaction, attention 
and memory. One of the most frequently used animal model of schizophrenia is the neonatal ventral hippocampus lesion $(\mathrm{nVHL})^{1,3}$. In the present mini-review, we discuss neurochemical and morphological changes in the PFC as reported in the nVHL rat.

\section{Prefrontal cortex connections in schizophrenia}

Numerous studies from different domains (clinical, postmortem, neuroanatomical and physiological) link the PFC with the pathophysiology of schizophrenia ${ }^{1,4}$. Indeed, the PFC is a complex region of sensorimotor and emotional integration, which participates in several cognitive functions such as attention processing, spatial memory, decision making etc. This cortical region receives glutamatergic projections from thalamus (dorsomedial nucleus), hippocampus and basolateral amygdala (BLA) ${ }^{1}$; dopaminergic projections from the ventral tegmental area (VTA); serotonergic projections from the median raphe nucleus; noradrenergic projections from locus coeruleus and cholinergic projections from basal forebrain. To close the circuit, PFC sends glutamatergic projections to nucleus accumbens (NAcc), CA1 region of the ventral hippocampus, BLA, VTA, and thalamus. Moreover, a recent report suggests that the connection among the $\mathrm{PFC}$, the lateral hypothalamus ( $\mathrm{LH}$ ) and the periaqueductal gray matter (PAG) together with the brainstem nuclei form a circuit that regulates the expression of positive emotions ${ }^{5}$. Interestingly, schizophrenic patients fail to express positive or negative emotions and are also unable to recognize emotions when presented with various faces ${ }^{6,7}$, hence it is possible to suggest that the PFC-LH-PAG circuit is dysfunctional in patients with schizophrenia.

It has been suggested that the connections between the thalamus and PFC are made during fifth month of gestation in humans, while the connections among the ventral hippocampus, BLA and PFC start to form at seventh month of gestation. Several reports suggest that the disruption between PFC and hippocampus at an early age is involved in the etiology of schizophrenia ${ }^{8-10}$. Rats that underwent nVHL at postnatal day (PND) 7 is an example of the early disruption between hippocampus and $\mathrm{PFC}^{1,3,8}$. Furthermore, this animal model shows behavioral, neurochemical and morphological changes that manifest mainly after puberty ${ }^{1,3,11}$.

\section{The nVHL Animal Model}

The nVHL rat is considered to be a neurodevelopmental model of schizophrenia ${ }^{1,3}$. This model was conceived by Lipska and Weinberger in the 1990's to address questions that pharmacological models could not answer ${ }^{12}$. First, the nVHL rat presents a disruption in the connections between the PFC and hippocampus in a critical postnatal period. Second, as stated in O'Donnell et al., ${ }^{13}$ "the periadolescent period is critical for maturation of PFC circuits" and recent research strongly suggests that adolescence is a critical period for functional organization in the PFC with high rate of synaptic pruning ${ }^{14}$. Indeed, juvenile nVHL rats present normal behaviors when compared to sham animals ${ }^{12,15}$ suggesting that the structural changes occur at this age. Third, after puberty, a constellation of behavioral, neurochemical and neuroanatomical changes are apparent in the nVHL rat ${ }^{1}$. The nVHL rat presents normal behaviors until the age of young adult as observed in schizophrenic patients. Therefore this animal model is suitable to further our understanding of this complex disorder.

\section{Neurochemical and neuromorphological changes in the nVHL rat}

The nVHL induces a constellation of neurochemical and neuronal alterations in the PFC as discussed below.

Neurochemical alterations: Overactivity of dopamine (DA) and glutamate in the mesolimbic system is the major working hypothesis of the etiology of schizophrenia ${ }^{1,16,17}$. In this regard, while Alquicer et al. (2004) ${ }^{18}$ observed reduced DA content in the PFC, exogenous application of SKF38393, a $\mathrm{D}_{1}$ agonist, increases cell firing of PFC pyramidal cells of the $n V H L$ rat $^{19}$. SKF38393 selectively increases the release of acetylcholine in the PFC of the nVHL rat at post-pubertal age $^{20}$. Whereas ventral tegmental area (VTA) stimulation increased cell firing in the PFC of the nVHL rat ${ }^{13}$. In a food paradigm in vivo, the nVHL rat maintains DA outflow for a longer period of time in the $\mathrm{PFC}^{21}$. Moreover, the nVHL rat presents enhanced sensibility to the DA agonist, amphetamine ${ }^{1}$. Regarding the glutamatergic system, the $\mathrm{nVHL}$ rats present hyperresponsiveness to MK-801, an n-methyl-D-Aspartate (NMDA) glutamatergic antagonist, showing no apparent changes in the expression of glutamatergic receptors ${ }^{15}$ Moreover, bath applications of NMDA also increases cell firing in the PFC of the nVHL rat ${ }^{13}$. The present data show hyper- and hypo-activity of DA in the PFC of the nVHL rat is observed most likely derived from contextual factors. Therefore, abnormal DA and glutamate systems alter the response to environmental or pharmacological challenges in the nVHL rat.

The inhibitory aminoacid gamma-aminobutyric acid (GABA) is consistently reduced in cortical areas in postmortem brains of schizophrenic patients ${ }^{22}$. Moreover, an abnormal balance between excitation and inhibition is commonly observed in psychiatric disorders ${ }^{17}$. In clear contrast, imaging studies have shown mixed results (increased, decreased or unaltered GABA levels ${ }^{23}$ ). Despite of the importance of GABA in the PFC, investigations of the levels or activity of GABA in the PFC of the nVHL rat has been minimal. Recently, Ryan et al. ${ }^{24}$ showed no significant difference in vesicular GABA transporter in the PFC of adult $\mathrm{nVHL}$ rat. However, further research is certainly warranted. 
Nitric oxide (NO) is an inter- and intra-cellular messenger ${ }^{25}$ involved in physiological processes including synaptic and neuronal plasticity ${ }^{26}$. Moreover, schizophrenic subjects present increased level of plasma $\mathrm{NO}^{27}$. The $\mathrm{nVHL}$ rat also presents increased levels of $\mathrm{NO}^{28}$ as well as increased nitric oxide synthase (NOS) immunostaining in the $\mathrm{PFC}^{29}$. In fact, NO has emerged as a key player to understand the neurochemical abnormalities observed in the nVHL rat. It has been established that NO interacts with DA and glutamate neurotransmitters ${ }^{30}$.

Animals that underwent the nVHL also present dysregulated proteins such as clathrin light chain $B$, a protein important for the expression of synaptophysin after puberty ${ }^{31}$; low level of nerve growth factor inducible-B $\mathrm{mRNA}^{32}$ and reduced expression of brain-derived neurotrophic factor (BDNF) $\mathrm{mRNA}^{33}$. In apparent agreement, patients with schizophrenia also exhibit these neurochemical alterations in the PFC including low expression of synaptophysin ${ }^{34}$ and decreased immunoreactivity to BDNF ${ }^{35,36}$. Consequently, all these neurotransmitters, growth factors and proteins have an impact on the synaptic connections that ultimately reshape neurons in the PFC.

\section{Neuromorphological alterations}

In postmortem brains of patients with schizophrenia, Garey et al..$^{37}$ observed reduced spine number in PFC layer III pyramidal neurons. While Koleske ${ }^{38}$ indicated that the shape of dendritic arbor determines the number and distribution of receptive synaptic contacts; Fiala et al., ${ }^{39}$ suggested that the dendritic spines are the main sites of excitatory input. Therefore alterations in spine density or dendritic arbor are associated with gain or loss of connectivity. Our group has shown a decrease in dendritic length and dendritic spines not only in the PFC layer V but also layer III in the nVHL rat ${ }^{28,40,41}$. The neuronal hypotrophy observed in PFC neurons has been associated with lack of input from the ventral hippocampus at a critical stage of development ${ }^{1}$.

Earlier disconnection (PD7 - PD9) of the ventral hippocampus and PFC pathway causes behavioral, neurochemical and neuromorphological changes after puberty or in early adulthood, which does not manifest itself if the damage had occurred before PD $14^{42}$. All this implies that there is a window of time in which the injury of this pathway may cause permanent damage. Moreover, the neonatal lesion of PFC, also causes changes in behavior, neurochemistry and morphology ${ }^{40,43-45}$, however these changes are not as copious as in the case of nVHL animals. Interestingly, at adult age, lesion of the PFC in nVHL animals, ameliorate behavioral changes ${ }^{46}$. Moreover, earlier lesion of $\mathrm{VH}$ altered the physiological response of PFC pyramidal neurons after puberty, which exhibited excessive firing in response to mesocortical stimulation ${ }^{13}$. Therefore, earlier disruption of the VH and PFC pathway affected the function of PFC after puberty, such as reduced levels of the BDNF in the PFC of the nVHL rats ${ }^{4}$.

\section{Conclusions}

The nVHL rat presents neurochemical and neuroanatomical alterations in the PFC similar to those observed in schizophrenic patients. Early on, O'Donnell ${ }^{48}$ stated that "disinhibited PFC could be responsible for cognitive deficits observed in schizophrenia". Moreover, PFC refinement occurs during late adolescence ${ }^{49}$ and the behavioral deficits in this animal model are also observed only after this period. Further investigations in this animal model are required to understand the pathology of this devastating disorder as well as to test potential novel drugs for the treatment of different deficits observed in schizophrenia.

\section{Acknowledgements}

GF and JCMM acknowledge the membership of National Research System of Mexico. JCMM is a recipient of International Brain Research Organization Return Home fellowship. We thank Mira Thakur for proofreading the manuscript.

\section{References}

1. Flores G, Morales-Medina JC, Diaz A. Neuronal and brain morphological changes in animal models of schizophrenia. Behavioural Brain Res. 2016; 301:190-203

2. Glantz LA, Lewis DA. Decreased dendritic spine density on prefrontal cortical pyramidal neurons in schizophrenia. Arch Gen Psychiatry. 2000; 57:65-73.

3. Tseng KY, Chambers RA, Lipska BK. The neonatal ventral hippocampal lesion as a heuristic neurodevelopmental model of schizophrenia. Behavioural Brain Res. 2009; 204:295-305.

4. Weinberger DR, Aloia MS, Goldberg TE, Berman KF. The frontal lobes and schizophrenia. J Neuropsychiatry \& Clinical Neurosci. 1994; 6:419-427.

5. Alvarez-Bolado G, Celio MR. The ventrolateral hypothalamic area and the parvafox nucleus: Role in the expression of (positive) emotions? J Comp Neurol. 2016; 524:1616-1623.

6. Mueser KT, Penn DL, Blanchard JJ, Bellack AS. Affect recognition in schizophrenia: a synthesis of findings across three studies. Psychiatry. 1997; 60:301-308.

7. Bortolon C, Capdevielle D, Raffard S. Face recognition in schizophrenia disorder: A comprehensive review of behavioral, neuroimaging and neurophysiological studies. Neurosci Biobehav Rev. 2015; 53:79-107

8. Tseng KY, Lewis BL, Lipska BK, O’Donnell P. Post-pubertal disruption of medial prefrontal cortical dopamine-glutamate interactions in a developmental animal model of schizophrenia. Biol Psychiatry. 2007; 62:730-738.

9. Belujon P, Patton MH, Grace AA. Role of the prefrontal cortex in altered hippocampal-accumbens synaptic plasticity in a developmental animal model of schizophrenia. Cereb Cortex. 2014; 24:968-977

10.Banks PJ, Burroughs AC, Barker GR, Brown JT, Warburton EC, Bashir ZI. Disruption of hippocampal-prefrontal cortex activity by dopamine D2R-dependent LTD of NMDAR transmission. Proc Natl Acad Sci U S A. 2015; 112:11096-11101 
11. Flores G, Atzori M. The Potential of Cerebrolysin in the Treatment of Schizophrenia. Pharmacol. Pharm. 2014; 5, 691-704.

12. Lipska BK, Weinberger DR. To model a psychiatric disorder in animals: schizophrenia as a reality test. Neuropsychopharmacology. 2000; 23:223-239.

13. O'Donnell P, Lewis BL, Weinberger DR, Lipska BK. Neonatal hippocampal damage alters electrophysiological properties of prefrontal cortical neurons in adult rats. Cereb Cortex. 2002; 12:975982.

14. Manitt C, Mimee A, Eng C, et al. The netrin receptor DCC is required in the pubertal organization of mesocortical dopamine circuitry. J Neurosci. 2011; 31:8381-8394.

15. Al-Amin HA, Shannon Weickert C, Weinberger DR, Lipska BK. Delayed onset of enhanced MK-801-induced motor hyperactivity after neonatal lesions of the rat ventral hippocampus. Biological Psychiatry. 2001; 49:528-539.

16. Howes 0 , McCutcheon R, Stone J. Glutamate and dopamine in schizophrenia: an update for the 21st century. J Psychopharmacol. $2015 ; 29: 97-115$

17. Grace AA. Dysregulation of the dopamine system in the pathophysiology of schizophrenia and depression. Nat Rev Neurosci. 2016; In press

18. Alquicer G, Silva-Gomez AB, Peralta F, Flores G. Neonatal ventral hippocampus lesion alters the dopamine content in the limbic regions in postpubertal rats. Int J Dev Neurosci. 2004; 22:103-111.

19. Tseng KY, O’Donnell P. Dopamine-glutamate interactions controlling prefrontal cortical pyramidal cell excitability involve multiple signaling mechanisms. J Neurosci. 2004; 24:5131-5139.

20. Laplante F, Srivastava LK, Quirion R. Alterations in dopaminergic modulation of prefrontal cortical acetylcholine release in postpubertal rats with neonatal ventral hippocampal lesions. J Neurochem. 2004; 89:314-323

21. Macedo CE, Angst MJ, Gobaille S, Schleef C, Guignard B, Guiberteau T, Louilot A, Sandner G. Prefrontal dopamine release and sensoryspecific satiety altered in rats with neonatal ventral hippocampal lesions. Behav Brain Res. 2012; 231:97-104.

22. Taylor SF, Tso IF. GABA abnormalities in schizophrenia: a methodological review of in vivo studies. Schizophr Res. 2015; 167:84-90.

23. Chiapponi C, Piras F, Caltagirone C, Spalletta G. GABA System in Schizophrenia and Mood Disorders: A Mini Review on ThirdGeneration Imaging Studies. Frontiers Psychiatry. 2016; 7:61.

24. Ryan RT, Bhardwaj SK, Tse YC, Srivastava LK, Wong TP. Opposing alterations in excitation and inhibition of layer 5 medial prefrontal cortex pyramidal neurons following neonatal ventral hippocampal lesion. Cereb Cortex. 2013; 23:1198-1207

25. Garthwaite J, Charles SL, Chess-Williams R. Endothelium-derived relaxing factor release on activation of NMDA receptors suggests role as intercellular messenger in the brain. Nature. 1988; 336:385-388.

26. Prast $\mathrm{H}$, Philippu A. Nitric oxide as modulator of neuronal function. Prog Neurobiol. 2001; 64:51-68.

27. Yanik $M$, Vural $H$, Kocyigit $A$, et al. Is the arginine-nitric oxide pathway involved in the pathogenesis of schizophrenia? Neuropsychobiology. 2003; 47:61-65.

28. Bringas ME, Morales-Medina JC, Flores-Vivaldo Y, et al. Clozapine administration reverses behavioral, neuronal, and nitric oxide disturbances in the neonatal ventral hippocampus rat. Neuropharmacology. 2012; 62:1848-1857.

29. Bernstein HG, Grecksch G, Becker A, Hollt V, Bogerts B. Cellular changes in rat brain areas associated with neonatal hippocampal damage. Neuroreport. 1999; 10:2307-2311.
30. Bernstein HG, Keilhoff G, Steiner J, Dobrowolny H, Bogerts B. Nitric oxide and schizophrenia: present knowledge and emerging concepts of therapy. CNS Neurol Disord Drug Targets. 2011; 10:792-807.

31.Vercauteren FG, Flores G, Ma W, et al. An organelle proteomic method to study neurotransmission-related proteins, applied to a neurodevelopmental model of schizophrenia. Proteomics. 2007; 7:3569-3579.

32.Bhardwaj SK, Beaudry G, Quirion R, Levesque D, Srivastava LK. Neonatal ventral hippocampus lesion leads to reductions in nerve growth factor inducible-B mRNA in the prefrontal cortex and increased amphetamine response in the nucleus accumbens and dorsal striatum. Neuroscience. 2003; 122:669-676.

33.Lipska BK, Khaing ZZ, Weickert CS, Weinberger DR. BDNF mRNA expression in rat hippocampus and prefrontal cortex: effects of neonatal ventral hippocampal damage and antipsychotic drugs. Eur J Neurosci. 2001; 14:135-144.

34.Glantz LA, Lewis DA. Reduction of synaptophysin immunoreactivity in the prefrontal cortex of subjects with schizophrenia. Regional and diagnostic specificity. Arch Gen Psychiatry. 1997; 54:660-669.

35.Ray MT, Shannon Weickert C, Webster MJ. Decreased BDNF and TrkB mRNA expression in multiple cortical areas of patients with schizophrenia and mood disorders. Transl Psychiatry. 2014; 4:e389.

36. Reinhart V, Bove SE, Volfson D, Lewis DA, Kleiman RJ, Lanz TA. Evaluation of TrkB and BDNF transcripts in prefrontal cortex, hippocampus, and striatum from subjects with schizophrenia, bipolar disorder, and major depressive disorder. Neurobiol Dis. 2015; 77:220-227.

37.Garey LJ, Ong WY, Patel TS, et al. Reduced dendritic spine density on cerebral cortical pyramidal neurons in schizophrenia. J Neurol Neurosurg Psychiatry. 1998; 65:446-453.

38. Koleske AJ. Molecular mechanisms of dendrite stability. Nat Rev Neurosci. 2013; 14:536-550.

39.Fiala JC, Spacek J, Harris KM. Dendritic spine pathology: cause or consequence of neurological disorders? Brain research Brain Res Rev. 2002; 39:29-54

40. Flores G, Alquicer G, Silva-Gómez AB, et al. Alterations in dendritic morphology of prefrontal cortical and nucleus accumbens neurons in post-pubertal rats after neonatal excitotoxic lesions of the ventral hippocampus. Neuroscience. 2005; 133:463-470.

41.Vazquez-Roque RA, Ramos B, Tecuatl C, et al. Chronic administration of the neurotrophic agent cerebrolysin ameliorates the behavioral and morphological changes induced by neonatal ventral hippocampus lesion in a rat model of schizophrenia. Journal of Neuroscience Res. 2012; 90:288-306.

42. Wood GK, Liang JJ, Flores G, Ahmad S, Quirion R, Srivastava LK. Cloning and in situ hybridization analysis of the expression of polysialyltransferase mRNA in the developing and adult rat brain. Brain Res Mol Brain Res. 1997; 51:69-81.

43. Flores G, Barbeau D, Quirion R, Srivastava LK. Decreased binding of dopamine D3 receptors in limbic subregions after neonatal bilateral lesion of rat hippocampus. J Neurosci. 1996; 16:2020-2026.

44.Brake WG, Flores G, Francis D, Meaney MJ, Srivastava LK, Gratton A. Enhanced nucleus accumbens dopamine and plasma corticosterone stress responses in adult rats with neonatal excitotoxic lesions to the medial prefrontal cortex. Neuroscience. 2000; 96:687-95.

45.Lazcano Z, Solis O, Díaz A, Brambila E, Aguilar-Alonso P, Guevara J, et al. Dendritic morphology changes in neurons from the ventral hippocampus, amygdala and nucleus accumbens in rats with neonatal lesions into the prefrontal cortex. Synapse. 2015; 69:314-25

46.Goto Y, O'Donnell P. Prefrontal lesion reverses abnormal mesoaccumbens response in an animal model of schizophrenia. Biol Psychiatry. 2004; 55:172-6. 
47. Molteni R, Lipska BK, Weinberger DR, Racagni G, Riva MA Developmental and stress-related changes of neurotrophic factor gene expression in an animal model of schizophrenia. Mol Psychiatry. 2001; 6:285-292.

48. O'Donnell P. Cortical disinhibition in the neonatal ventral hippocampal lesion model of schizophrenia: new vistas on possible therapeutic approaches. Pharmacol Ther. 2012; 133:19-25.

49. Manitt C, Eng C, Pokinko M, Ryan RT, Torres-Berrio A, Lopez JP, et al. dcc orchestrates the development of the prefrontal cortex during adolescence and is altered in psychiatric patients. Transl Psychiatry. 2013; 3:e338. 